\title{
Strategyproof Facility Location with Concave Costs
}

\author{
DIMITRIS FOTAKIS \\ National Technical University of Athens \\ and \\ CHRISTOS TZAMOS \\ Massachusetts Institute of Technology
} In $k$-Facility Location games, $n$ strategic agents report their locations on the real line and a
mechanism maps them to $k$ facilities. Each agent seeks to minimize her connection cost to the
nearest facility and the mechanism should be strategyproof and approximately efficient. Facility
Location games have received considerable attention in the framework of approximate mechanism
design without money. In this letter, we discuss some recent positive results on the approximability
of $k$-Facility Location by randomized strategyproof mechanisms. Interestingly, these results hold
even if the agents' connection cost is a concave cost function of the distance.

Categories and Subject Descriptors: F.2 [Theory of Computation]: Analysis of Algorithms and Problem Complexity; J.4 [Computer Applications]: Social and Behavioral SciencesEconomics

General Terms: Algorithms, Theory, Economics

Additional Key Words and Phrases: Algorithmic Mechanism Design, Facility Location Games

\section{INTRODUCTION}

Facility Location games provide an elegant model of collective decision making when the outcome consists of $k \geq 1$ components (e.g., public facility locations, political representatives), each agent is interested in her most preferable component, and the agents have a structured and homogeneous view towards different alternatives, usually quantified by an embedding of the alternatives in a metric space. Due to the rich additional structure in the domain, several classes of Facility Location games escape the impossibility result of Gibbard-Satterthwaite and allow for interesting and socially efficient strategyproof mechanisms. A classical result in this direction concerns the choice of a location on the real line based on single-peaked preferences. Moulin [1980] proved that the class of deterministic strategyproof mechanisms for this problem coincides with the class of generalized median mechanisms. Subsequently, Schummer and Vohra [2002] extended this characterization to tree networks, but also proved that for non-tree networks, any onto strategyproof mechanism is a dictatorship.

To circumvent similar impossibility results applying to more complex (and more interesting) cases of Facility Location games, researchers have recently adopted an optimization viewpoint, originally suggested by Procaccia and Tennenholtz [2009]. The general idea is to consider the well known optimization problem of $k$-Facility Location and investigate the best approximation ratio achievable by (deterministic or randomized) strategyproof mechanisms.

Authors' addresses: fotakis@cs.ntua.gr, tzamos@mit.edu 


\subsection{The Model and Related Work}

Along these lines, we consider $k$-Facility Location on the line, where $k$ locations are chosen based on the preferences of $n$ strategic agents. Each agent $i$ has an ideal location $x_{i} \in \mathbb{R}$, which is private information, and a mechanism maps the agents' reported locations to a vector $\vec{z}=\left(z_{1}, \ldots, z_{k}\right)$ of $k$ facility locations. Each agent $i$ evaluates the outcome according to her connection cost, given by a nonnegative and nondecreasing function $c\left(d_{i}\right)$ of the distance $d_{i}=\min _{j \in[k]}\left|x_{i}-z_{j}\right|$ of $i$ 's true ideal location to the nearest facility. The agents seek to minimize their connection cost and may misreport their ideal locations in an attempt to manipulate the mechanism. Hence, the mechanism should be strategyproof, i.e., should ensure that no agent can improve her connection cost by misreporting her location.

The goal of the society is to minimize a function of the agents' connection cost. Most prominent among them are the objective of SocIAL CosT, which considers the total connection cost of the agents, and the objective of MAx CosT, which considers the maximum connection cost of an agent. So, in addition to the essential requirement of strategyproofness, the mechanism should either optimize or achieve a reasaonable approximation ratio to the designated objective function, thus ensuring that the outcome is socially efficient (or at least, tolerable).

Recently there has been a considerable interest in quantifying the best approximation ratio achievable by strategyproof mechanisms for $k$-Facility Location when the agents' connection cost is linear in their distance to the nearest facility. The main message is that deterministic strategyproof mechanisms can achieve a bounded approximation ratio ${ }^{1}$ only if we have at most 2 facilities [Procaccia and Tennenholtz 2009; Fotakis and Tzamos 2013a]. On the other hand, randomized mechanisms are known to achieve better approximation ratios for 2 facilities and also a bounded approximation ratio if we have any number $k$ of facilities and only $k+1$ agents [Escoffier et al. 2011]. Notably, instances with only $k+1$ agents are known to be hard for deterministic mechanisms.

\section{THE EQUAL-COST MECHANISM}

In [Fotakis and Tzamos 2013b], we present a simple randomized mechanism, the so-called EQUAL-COST, which applies to instances with any number $k$ of facilities and any number $n$ of agents, and is the first known strategyproof mechanism with a bounded approximation ratio for all $k$ and $n$.

EQUAL-COST works by equalizing the expected connection cost of all agents, and essentially achieves strategyproofness by fairness. For simplicity, let us first focus on the identity cost function $c(d)=d$. Then, given the number $k$ of facilities and the vector $\vec{x}=\left(x_{1}, \ldots, x_{n}\right)$ of the agents' reported locations:

Step 1. EQUAL-Cost computes an optimal covering of all agent locations with $k$ disjoint intervals $\left[\alpha_{j}, \alpha_{j}+\ell\right]$ that minimizes the common interval length $\ell$.

Step 2. Let $X$ be a random variable taking on the values of 0 and $\ell$ equiprobably.

Step 3. For every interval $\left[\alpha_{j}, \alpha_{j}+\ell\right]$, EQUAL-Cost places a facility at $\alpha_{j}+X$, if $j$ is odd, and at $\alpha_{j}+\ell-X$, if $j$ is even.

\footnotetext{
${ }^{1}$ The approximation ratio of a $k$-Facility Location mechanism is bounded if it only depends on $n$,
} $k$, and possibly $c$. We note that this property is essentially objective-independent. 
We can efficiently compute, in step 1 , a covering of $\vec{x}$ with $k$ disjoint intervals of minimum length $\ell$. By the definition of $\ell$, the optimal maximum cost is $\ell / 2$. Since EQUAL-Cost places a facility at each interval, the distance of any agent to the nearest facility is at most $\ell$. This implies an approximation ratio of 2 for the objective of MAX Cost and an approximation ratio of $n$ for the Social Cost.

EQUAL-CosT ensures that the expected connection cost of every agent is equal to $\ell / 2$ and independent of her location. This holds because if an agent has connection $\operatorname{cost} \delta$ when the facility is located at the left endpoint of her interval, her connection cost is $\ell-\delta$ when the facility is located at the right endpoint. Since, in step 2, we choose among the two outcomes equiprobably, the expected cost of the agent is $\ell / 2$, i.e., independent of her location. We also note here that the correlation in the facility placement, introduced in step 3 , is needed to guarantee that no agent can get a lower connection cost because of a facility in a different interval.

Intuitively, strategyproofness follows because every agent is only interested in the interval length $\ell$, and EQUAL-CosT makes $\ell$ as small as possible. Therefore, it is not profitable for an agent to misreport her location and increase $\ell$. Moreover, even if an agent reports a false location that causes the interval length to decrease from $\ell$ to $\ell^{\prime}$, the distance of her true location to the nearest interval is at least $\ell-\ell^{\prime}$. Hence, the expected connection cost of any liar is always greater than $\ell / 2$, which implies that EQUAL-COST is strategyproof (and also group strategyproof).

The properties of EQUAL-COST reveal an interesting separation between deterministic strategyproof mechanisms, whose approximation ratio for MAX CosT jumps from 2 to unbounded when $k$ increases from 2 to 3 , and randomized strategyproof mechanisms, whose approximation ratio remains at most 2 for all $k$.

Moreover, for any given concave cost function $c(d)$, we can construct, in step 2, a random variable $X$, taking on values in $[0, \ell]$, such that all locations covered by an interval have the same expected connection cost $\mathbb{E}[c(X)]$. With such a random variable $X$ in step 3, EQUAL-CosT retains the properties outlined above, including group strategyproofness and the approximation guarantees. On the negative side, a similar result is not possible for the class of all convex cost functions, in the sense that there are convex cost functions $c$ for which the approximation ratio of any randomized strategyproof mechanism cannot be bounded in terms of $n, k$, and $c$.

Considering arbitrary concave cost functions, we not only show that good randomized strategyproof approximations are possible for a more general setting of $k$-Facility Location, but also take a first step towards closing the gap between linear connection costs, which are common in Combinatorial Optimization, and the general setting of single-peaked preferences, which is standard in Social Choice. The goal would be to have mechanisms that are strategyproof for any (even unknown) cost function $c$ in a large class of functions, just as generalized medians are strategyproof for any collection of single-peaked preferences, while the approximation ratio may depend on some quantitative properties (e.g., the derivative) of $c$.

\section{THE PICK-THE-LOSER MECHANISM}

EQUAL-COST performs quite well for the objective of MAX COST, but may perform poorly for the objective of Social Cost. E.g., in the extreme case of $n=k+1$ agents, where one can easily satisfy all but one of them, EQUAL-CosT imposes an 
unreasonably high expected connection cost on all agents for the sake of fairness.

In [Fotakis and Tzamos 2013b], we present a natural randomized mechanism, the so-called PICK-THE-Loser, that applies to instances with only $k+1$ agents and achieves a good approximation ratio for the objective of Social Cost. The idea is to place a facility at the locations of all agents but one, who is designated as the loser. The Social Cost is minimized if the agent with the minimum connection cost (given that all other agents get a facility) is the loser. Since such a deterministic greedy selection is not strategyproof, we select the loser in a randomized way that balances the bias towards small connection costs against strategyproofness. Specifically, given the cost function $c$ and the agents' reported locations $\vec{x}=\left(x_{1}, \ldots, x_{n}\right)$, where $x_{1}<x_{2}<\cdots<x_{n}$, PICK-THE-LOSER works as follows:

Step 1. For each even-numbered agent $i$, it selects $s_{i} \in(0,1)$ uniformly at random and computes $i$ 's scaled connection cost $c_{i}=\min _{j \neq i} c\left(\left|x_{j}-x_{i}\right|\right) / s_{i}$.

Step 2. The even-numbered agent with the minimum scaled connection cost is designated as the loser. A facility is placed at the locations of all other agents.

We note that PICK-THE-LOSER treats odd-numbered and even-numbered agents differently. The intuition is that after allocating a facility to all odd-numbered agents, the facility allocation to even-numbered agents can be regarded as an allocation of $n^{\prime}=\lfloor n / 2\rfloor-1$ identical private goods to $n^{\prime}$ agents, where the connection costs correspond to the agent valuations.

For any given concave cost function $c$, PICK-THE-LOSER is group strategyproof and achieves an approximation ratio of 2 for the objective of SocIAL COST and an approximation ratio of 4 for the objective of MAX Cost. Furthermore, this result suggests that the idea of random uniform cost scaling may be useful in other applications where we seek a good strategyproof approximation to the minimum cost agent.

\section{REFERENCES}

Escoffier, B., Gourvìs, L., Thang, N., Pascual, F., and Spanjaard, O. 2011. Strategy-proof mechanisms for Facility Location games with many facilities. In Proc. of the 2nd International Conference on Algorithmic Decision Theory (ADT '11), LNAI 6992, 67-81.

Fotakis, D. And Tzamos, C. 2013a. On the power of deterministic mechanisms for Facility Location games. In Proc. of the 40th International Colloquium on Automata, Languages and Programming (ICALP '13), LNCS 7965, 435-452.

Fotakis, D. And Tzamos, C. 2013b. Strategyproof Facility Location with concave costs. In Proc. of the 14th ACM Conference on Electronic Commerce (EC '13), 435-452.

Moulin, H. 1980. On strategy-proofness and single-peakedness. Public Choice 35, 437-455.

Procaccia, A. And Tennenholtz, M. 2009. Approximate mechanism design without money. In Proc. of the 10th ACM Conference on Electronic Commerce (EC '09), 177-186.

Schummer, J. And VohrA, R. 2002. Strategyproof location on a network. Journal of Economic Theory 104, 405-428. 